\title{
Structure of high and low shear-stress events in a turbulent boundary layer
}

\author{
G. Gomit, R. de Kat and B. Ganapathisubramani * \\ Engineering and the environment, University of Southampton, Southampton, \\ SO17 1BJ, UK
}

\begin{abstract}
Simultaneous particle image velocimetry (PIV) and wall-shear-stress sensor measurements were performed to study structures associated with shear-stress events in a flat plate turbulent boundary layer at a Reynolds number $R e_{\tau} \approx 4000$. The PIV field-of-view covers $8 \delta$ (where $\delta$ is the boundary layer thickness) along the streamwise direction and captures the entire boundary layer in the wall-normal direction. Simultaneously, wall-shear-stress measurements that capture the large-scale fluctuations were taken using a spanwise array of hot-film skin-friction sensors (spanning $2 \delta$ ). Based on this combination of measurements, the organization of the conditional wall-normal and streamwise velocity fluctuations $(u$ and $v$ ) and of the Reynolds shear-stress $(-u v)$ can be extracted. Conditional averages of the velocity field are computed by dividing the histogram of the large-scale wall-shear-stress fluctuations into four quartiles, each containing $25 \%$ of the occurrences. The conditional events corresponding to the extreme quartiles of the histogram (positive and negative) predominantly contribute to a change of velocity profile associated with the large structures and in the modulation of the small-scales. A detailed examination of the Reynolds shear-stress contribution related to each of the four quartiles shows that the flow above a low wall-shearstress event carries a larger amount of Reynolds shear-stress than the other quartiles. The contribution of the small- and large-scales to this observation is discussed based on a scale decomposition of the velocity field.
\end{abstract}

\section{Introduction}

Over the last few decades, a number of studies have focused on characterisation and on understanding the large structures present in the outer region of a turbulent boundary layer. The presence of these large structures was initially revealed using two point correlations based on hot-wire measurements (Kovasznay et al. 1970; Blackwelder and Kovasznay (1972); Brown and Thomas (1977); Bandyopadhyay and Hussain (1984); Wark and Nagib (1991)). These studies revealed the presence of low frequency content in the log region from statistics of fluctuating streamwise velocity signals. More recently, optical methods such as the particle image velocimetry (PIV), in planes parallel to the wall, allowed the observation of these coherent structures in the form of elongated and meandering narrow strips of uniform momentum (Ganapathisubramani et al. (2003), Tomkins and Adrian (2003)). These elongated structures have also been

*G.Bharath@soton.ac.uk 
observed in pipe flows and labeled very-large-scale motions (VLSM) by Kim and Adrian (1999), or in channel flows based on DNS data (Del Alamo et al. (2004)).

Using hot-wire rake measurements, Hutchins and Marusic (2007a) confirmed that very long meandering features populate the log region of turbulent boundary layers, extending to over $20 \delta$ in length and carry a large proportion of the Reynolds stress in the log region. They named these events "superstructures". Their spectral analysis suggests that the peak in the premultiplied spectra in the outer region of a boundary layer could be caused by these large-scale structures. Moreover, these very large-scale structures maintain a footprint at the wall. The existence of this footprint has been also noted in Schlatter et al. (2009).

The magnitude of the footprint of the superstructures at the wall increases with increasing Reynolds number. This is not only in terms of a direct footprint (wall-shear-stress fluctuation) but also modulates the amplitude and frequency of the near-wall scales. This modulation is also present farther away from the wall, however, the nature of the modulation switches at some vertical position, which is near the middle of the log region. The modulation of small-scales by the large-scale events has been described and discussed in various studies Hutchins and Marusic (2007b); Mathis et al. (2009); Marusic et al. (2010); Schlatter and Örlü (2010); Bernardini and Pirozzoli (2011); Hutchins et al. (2011); Ganapathisubramani et al. (2012); Talluru et al. (2014); Agostini and Leschziner (2014); Agostini et al. (2016).

Pertinent to this study is the amplitude modulation effect that was based on conditional analysis using simultaneous wall-shear-stress and hot-wire measurement in Hutchins et al. (2011) and Talluru et al. (2014). In these two studies, an array of surface hot-films was used to detect the footprint of the VLSM and simultaneous hot-wire measurements were used to compute the 3D conditional structures associated with these events using Taylor's hypothesis. Hutchins et al. (2011) showed that for a negative wall-shear-stress event, the conditional mean streamwise velocity reveals the presence of a forward-leaning low-speed structure above the low skin-friction event bordered by two high-speed regions. The conditional values of small-scale turbulence intensity in the streamwise direction show that the low-speed structure is associated with a decrease of the small-scale activity near the wall, switching to an increase of this activity farther away from the wall. Talluru et al. (2014) expanded on this study by performing simultaneous wall-shear-stress and three component hot-wire measurements. Using conditional averages, they showed that a large-scale low-skin-friction event is formed between counter-rotating roll modes. Moreover, they observed that all the three small-scale components are modulated in a very similar way by large-scale streamwise fluctuations. Interestingly, they showed that the unfiltered Reynolds shear-stresses are also modulated in a very similar fashion by the large-scale events.

These previous studies decomposed the wall-shear-stress as positive and negative fluctuations and do not apply any further condition to it. This broad condition does not capture all the details of the interactions. Ganapathisubramani et al. (2012) examined amplitude modulation using velocity signals and showed that for small-amplitude large-scale signal, the modulation effect was symmetric but asymmetries in the modulation appears for more extreme values of large-scale fluctuation. More recently, Agostini et al. (2016) also used velocity signals and showed that the amplitude modulation effect is in fact different for extreme positive and negative velocity fluctuations. In this paper, we use simultaneous wall-shear-stress and wide-field velocity measurements and examine if this asymmetry in modulation extends to the wall. We perform conditional averaging, based on high and low large-scale shear-stress events, and examine the mean flow as well as the normal and Reynolds shear-stresses. 


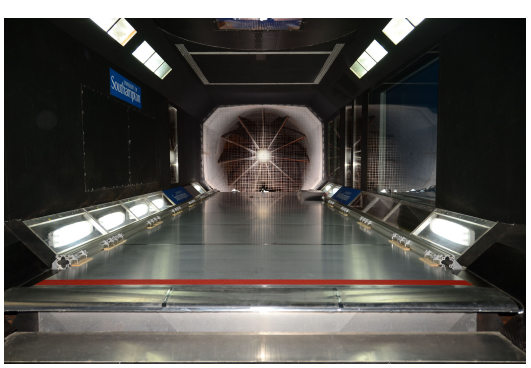

(a)

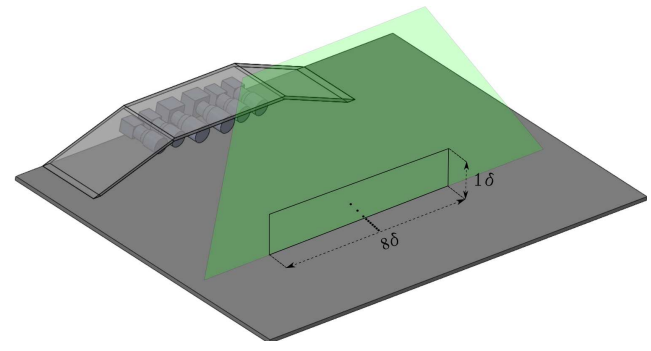

(c)

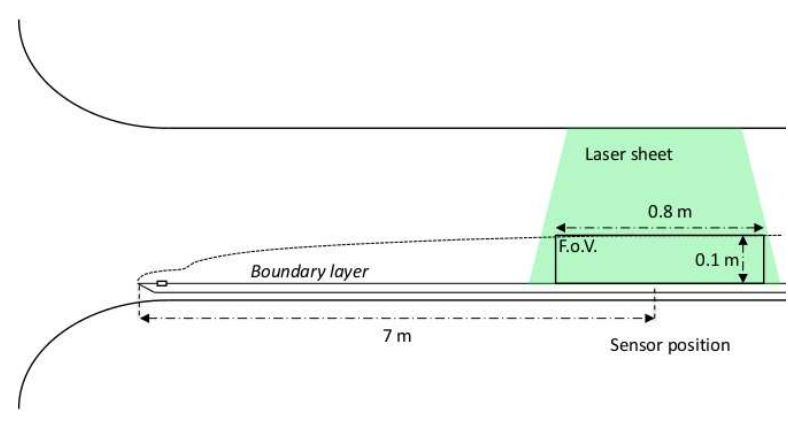

(b)

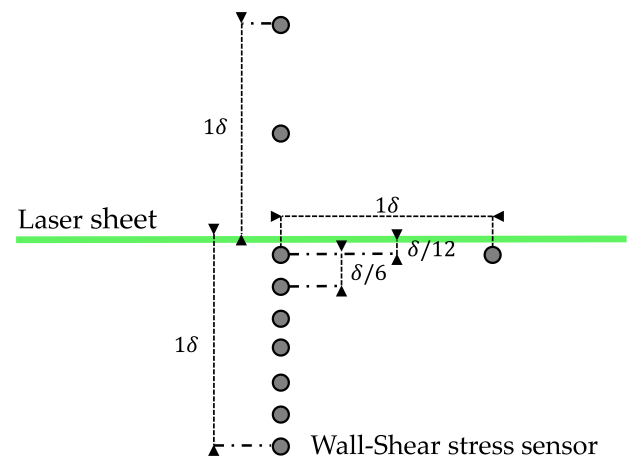

(d)

Figure 1: Experimental set-up: a) Photograph of the flat plate in the wind-tunnel, b) Schematic of the global experimental set-up (flow left to right), c) Schematic of the PIV set-up (flow right to left), d) Location of the wall-shear-stress sensors (flow left to right).

\section{Experimental methods}

The experiments were performed in the R.J. Mitchell wind tunnel of the University of Southampton (figure 1). This low speed closed-loop wind tunnel has a $10 \mathrm{~m}$ long test section with a cross section $3.4 \mathrm{~m}$ wide and $2.4 \mathrm{~m}$ high. The experiments were conducted with a free-stream velocity $U_{\infty}=15.8 \mathrm{~m} \cdot \mathrm{s}^{-1}$. The turbulent boundary layer develops over a flat plate installed along the length of the test section. The leading edge of the flat plate was shaped as a half ellipse. A 0.16 $\mathrm{m}$ long strip of rough sand paper was used to trigger transition to turbulence $0.4 \mathrm{~m}$ downstream the leading edge.

Simultaneous particle image velocimetry and hot-film shear-stress sensor measurements were performed approximately $7 \mathrm{~m}$ downstream from the leading edge, in the center plane of the cross section. The turbulent boundary layer thickness, $\delta$, was computed with the method proposed by Jones et al. (2001). At the center of the field of view, $\delta$ is equal to $0.1 \mathrm{~m}$. For comparison, the boundary layer thickness based on $U_{99}=0.99 U_{\infty}$, is $\delta_{99}=0.085 \mathrm{~m}$. Variations of the boundary layer thickness is less than $2 \%(2 \mathrm{~mm})$ across the field of view. The skin-friction velocity at the center of the field of view is $U_{\tau}=0.60 \mathrm{~m} \cdot \mathrm{s}^{-1}$. Variations of the order of $2 \%$ of $U_{\tau}$ are observed along the field of view. This variation in skin-friction is based on Clauser chart method applied to the mean profile and is therefore within the uncertainty of the method. The Reynolds number based on the friction velocity and boundary layer thickness is $R e_{\tau} \approx 4000$. 


$\begin{array}{cccccccc}x(\mathrm{~m}) & U_{\infty}\left(\mathrm{m} \cdot \mathrm{s}^{-1}\right) & \delta(\mathrm{m}) & \delta_{99}(\mathrm{~m}) & \theta(\mathrm{m}) & U_{\tau}\left(\mathrm{m} \cdot \mathrm{s}^{-1}\right) & R e_{\tau} & R e_{\theta} \\ 7 & 15.76 & 0.100 \pm 0.002 & 0.085 \pm 0.002 & 0.008 & 0.60 \pm 0.01 & 4000 \pm 150 & 8500\end{array}$

Table 1: Boundary layer parameters

Throughout this paper, $x, y$, and $z$ are the streamwise, wall-normal and spanwise directions, respectively, while $u$ and $v$ are the streamwise and wall-normal velocity fluctuations. The origin of the coordinate system in the streamwise direction corresponds to the wall-shear-stress sensors array position. In the spanwise direction, the laser sheet plane is located in the $x-y$-plane $z / \delta=0$. Time-averaged values are either denoted by upper case, as $U$, or overbar, as $\overline{u^{2}}$. Values that are conditionally averaged are denoted as $\langle u\rangle$. All the quantities that are normalized using $U_{\tau}$ and $\nu$ are denoted with a superscript + , such as $y^{+}=y U_{\tau} / \nu$.

The main parameters of the boundary-layer are summarized in table 1 and the overall mean and variance profiles are shown in figure 2. For comparison, data from Hutchins et al. (2009) (hot-wire data, similar $R e_{\tau}$ ) and Eitel-Amor et al. (2014) (numerical data, similar $R e \theta$ ) are included. In addition, Stereo-PIV data from Kähler et al. (2002) and Tomo-PIV data from Laskari et al. (2016) are included for validation. Mean and fluctuations of streamwise velocity are well captured and consistent trends are found for the wall-normal velocity fluctuations and Reynolds shear-stress. Due to laser reflections, no velocity data is available below $y^{+}=100$ for the current data set. The differences between the current PIV, the hot-wire data and the numerical results can be attributed to different spatial resolution of the experiments(Hutchins et al. 2009) as well as the different $R e_{\theta}$ and $R e_{\tau}$ between the datasets. Moreover, for turbulent flows, several studies including Willert and Gharib (1991) or Foucaut et al. (2004) have discussed the low-pass filtering effect of PIV, due to the averaging over the Interrogation Windows (IW). As mentioned by Kähler et al. (2012), in the case of near-wall flow, the nonuniform distribution of particles near the wall and the spatial low-pass filtering introduce a bias in the location of the correlation peak resulting in higher uncertainties. However, even if a filtering effect is present close to the wall, in the following sections the small scales are defined as scales with a size of about 100 wall units to $1 \delta$. The scales larger to $1 \delta$ are considered to be large-scales. Therefore, the observed discrepancies will not change the conclusions drawn from the analysis present in subsequent sections.

\subsection{PIV measurement}

A schematic of the PIV set-up is provided in figure 1c. The flow was seeded using a smoke machine that generates droplets with a mean diameter of $1 \mu \mathrm{m}$. The beams of two pulsed Nd-YAG lasers (532 nm, $200 \mathrm{~mJ}$ ) were combined using mirrors, a half-wave plate, and a highpower polarising beam splitter. A four lens system (two spherical and two cylindrical) was used simultaneously to create an extended laser sheet in the $x-y$ plane of the boundary layer. An array of six high resolution cameras were used: four Lavision, ImagerPro Lx 16Mpx and two Lavision, ImagerPro Lx 29Mpx. The $16 \mathrm{Mpx}$ cameras were equipped with Nikkor $200 \mathrm{~mm}$ lenses and the 29Mpx cameras with Sigma $105 \mathrm{~mm}$ lenses and Kenko $1.4 \times$ teleconverters. The cameras were protected from the flow and seeding by a parallelogram shaped aluminum box with a window for optical access. A schematic of the camera setup is shown in figure 1c. Each $29 \mathrm{Mpx}$ camera, located in the middle of the cameras array, captured $1.4 \delta(0.14 \mathrm{~m})$ in the wall-normal direction and $2.5 \delta(0.25 \mathrm{~m})$ in the streamwise direction. The $16 \mathrm{Mpx}$ cameras, placed on the edge of the cameras array, covered $1 \delta(0.1 \mathrm{~m})$ in the wall-normal direction and $1.5 \delta(0.15 \mathrm{~m})$ in the streamwise direction. The field of view of the entire camera array is $8 \delta \times \delta(0.8 \mathrm{~m} \times 0.1 \mathrm{~m})$ 


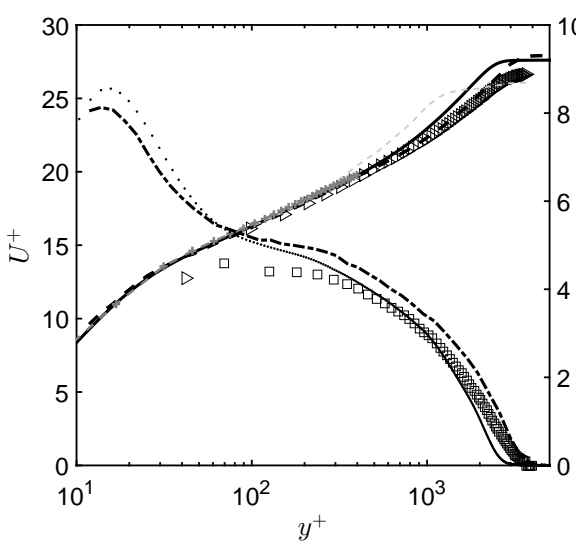

(a)

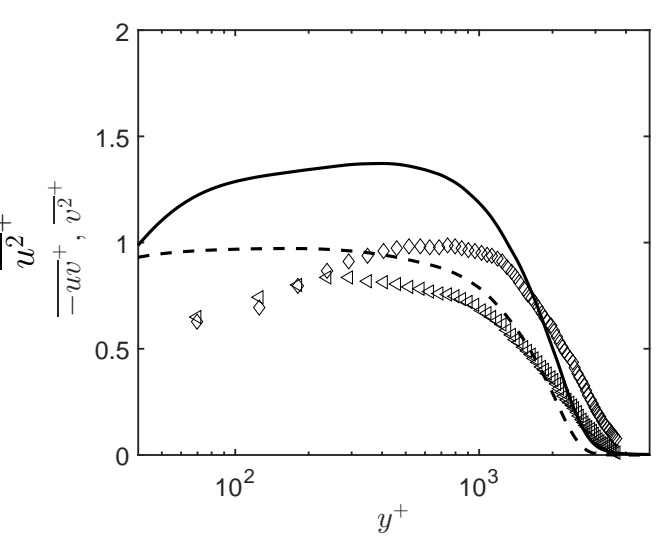

(b)

Figure 2: Statistical profiles of the turbulent boundary layer compared to data from Hutchins et al. (2009) (hot-wire data, $\left.R e_{\tau}=3910\right)$, Eitel-Amor et al. (2014) (LES data, $R e_{\theta}=8300$ ), Kähler et al. (2002) (Stereo-PIV, $\left.R e_{\theta}=8100\right)$ and Laskari et al. (2016) (Tomo-PIV, $R e_{\theta}=4850$ ) (a) Mean velocity profile $U^{+}$: right triangle (measured), solid line (LES data), dashed line (HW data), dashed gray line (Tomo-PIV), gray line with crosses (Stereo-PIV); $\bar{u}^{+}$: square (measured), dot-line (LES data) and line with cross (HW data); (b) ${\overline{v^{2}}}^{+}$: diamond (measured) and solid line (LES Data); Reynolds shear-stress, $-\overline{u v}^{+}$: left triangle (measured) and dashed line (LES data).

in the streamwise-wall-normal plane. The vector fields were computed on a coarse grid $(32 \times 32$ pixel interrogation area) with an initial particle image displacement offset and refined to a fine grid $(24 \times 24$ pixel interrogation area). The resulting spatial resolution of the measurement is approximately $l^{+}=55(1.4 \mathrm{~mm})$ and was sampled with a grid spacing of $h^{+}=15(0.36 \mathrm{~mm})$ by using an interrogation area overlap of $75 \%$. The acquisition frequency of the PIV system was $0.25 \mathrm{~Hz}$, which resulted in all velocity field representing independent samples. A total of 4000 instantaneous fields were recorded simultaneously with wall-shear-stress sensor measurements.

\section{$2.2 \quad$ Wall sensors}

Ten skin-friction sensors were used to capture the wall-shear-stress footprint of large-scale flow structures. The sensors operate in constant temperature mode. Each probe consists of $0.2 \mu \mathrm{m}$ thick, $0.1 \mathrm{~mm}$ long, $1.5 \mathrm{~mm}$ wide nickel wire, that corresponds to approximately $l_{w}^{+} \approx 60$, with copper leads deposited onto a polyamide substrate (Senflex ${ }^{\circledR}$ sensors from Tao Systems). This wire was then glued onto an alumina 2-bore cylindrical insulating support of $6 \mathrm{~mm}$ in diameter. Stainless steel needles were then inserted through the support and soldered onto the sensor's copper leads. The CTA system used was a Dantec miniCTA ${ }^{\circledR}$ operating with an overheat ratio of 1.5. The sensors were calibrated using mean velocity profiles measured with the PIV system at 5 different free stream velocities $\left(10,12.5,15,17.5\right.$ and $\left.20 \mathrm{~m} \cdot \mathrm{s}^{-1}\right)$. The friction velocity was computed using Clauser's method and interpolated using the expression proposed in Hutchins et al. (2011):

$$
\frac{U_{\tau}}{U_{\infty}}=\frac{1}{C_{1}+C_{2} \log R e_{x}}
$$


The CTA signals were acquired at a sampling frequency of $5 \mathrm{kHz}$ (antialiasing filter at 2.5 $\mathrm{kHz}$ ) and subsequently low-pass filtered at $500 \mathrm{~Hz}$ to retain only the large-scale component of the shear-stress. The shear-stress signal was recorded continuously during the PIV acquisition and the two measurement systems were synchronised to relate the friction velocity to the velocity field measured with the PIV.

The positions of the sensors relative to the laser sheet are given in figure 1d. The spanwise array is composed of nine sensors and covers $2 \delta$ in the spanwise direction. The first sensor is located on one side of the laser sheet at a distance of $0.08 \delta(8 \mathrm{~mm})$ from the laser sheet. Subsequent sensors are placed at a spacing of $0.16 \delta$ as shown in the figure. On the other side of the laser sheet, two sensors are located at $\mathrm{z}=0.56 \delta$ and $1.04 \delta$. These two sensors are used to confirm the statistical symmetry of the statistics. In addition to these, one sensor is placed downstream of the spanwise array such that two sensors are aligned in the streamwise direction and spaced by $1 \delta$.

The histogram of the velocity friction fluctuations measured with the sensors is presented in figure 3. Alfredsson et al. (1988) showed values of skewness between 0.3 to 1 and a $u_{\tau}^{r m s}$ between $15 \%$ and $40 \%$ of the mean value for experimental data and approximately $20 \%$ based on DNS data. The histogram of the current data set does not display a skewed distribution and the standard deviation of the signal obtained with the sensors, $u_{\tau}^{r m s}$, is around $8 \%$ of the mean value. These values are lower than found previously and is primarily due to the attenuation of the small-scale fluctuations of the friction velocity. This attenuation is primarily due to the frequency response of the glue-on skin-friction sensors, which was approximately $200 \mathrm{~Hz}$. As described in Alfredsson et al. (1988) and discussed in Hutchins et al. (2011), the frequency response of the sensor is reduced due to the thermal inertia of the substrate (on which the sensors are placed). However, the frequencies associated with the the large-scale events are lower than $125 \mathrm{~Hz}$ (this corresponds to structures that are at least $\delta$ long) and will be captured by the sensor.

It should be emphasized that the sensors are only used to detect and characterize the largescale events. The fact that the small-scale fluctuations are not captured by the sensors does not alter the results presented in the following sections. To validate this affirmation, the two-point correlation map computed from the sensor array is shown in figure 4. This result is consistent with the one presented in Hutchins et al. (2011). A region of elongated positive correlation is flanked on each side by an area of negative correlation. The distance between the centers of these negative regions is about $0.6 \delta$.

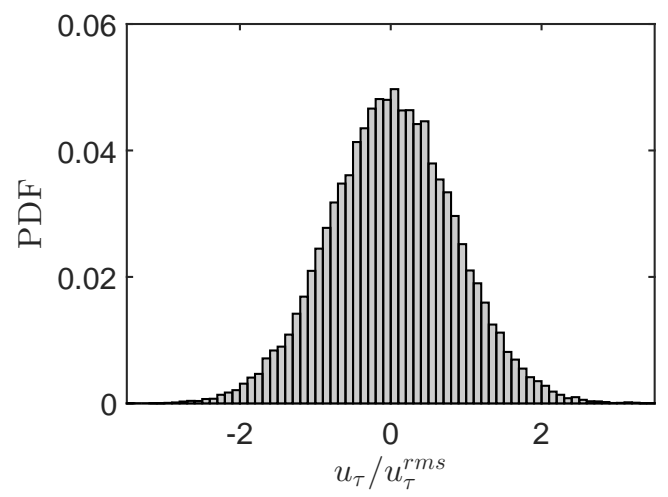

Figure 3: Histogram of the normalized fluctuations of the friction velocity. 


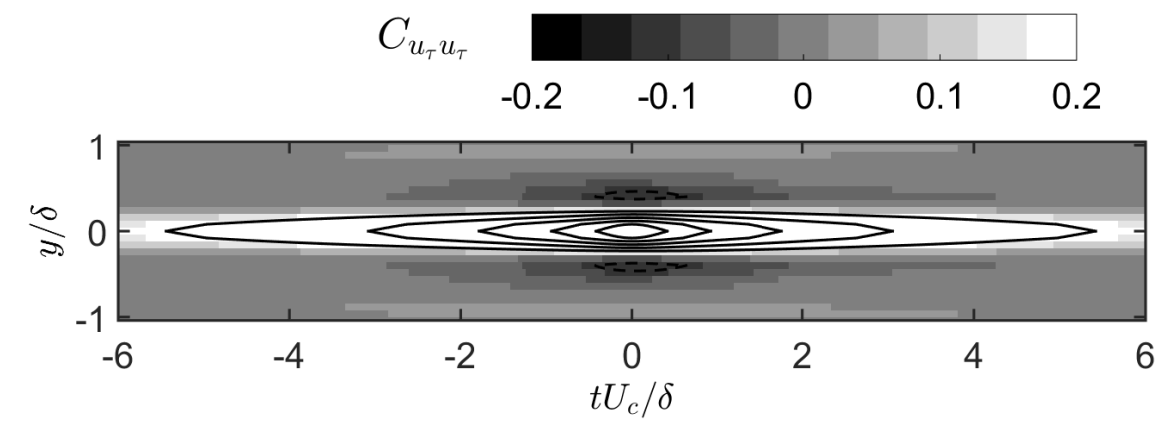

Figure 4: Two-point correlation map $C_{u_{\tau} u_{\tau}}$. The contour lines correspond to level 0.1 to 0.9 with 0.2 increment. The dashed lines are negative contour and solid contours are positive $C_{u_{\tau} u_{\tau}}$.

\subsection{Instantaneous view of large-scale structures}

The simultaneous PIV and shear-stress measurements can now be used for the characterisation of the flow structures related to low and high shear-stress events. Particularly, the frictionvelocity fluctuations were used to detect the footprint of the large-scale structures in a similar way as Talluru et al. (2014). The data from the sensors are filtered using a Gaussian filter with a standard deviation of about $0.5 \delta / U_{c}$ in the streamwise direction and $\delta / 6$ (2 adjacent sensors) in the spanwise direction in order to extract only the large-scale contribution of the skin-friction fluctuations. Note that $U_{c}$ is an approximate advection velocity that is used to map the timeseries of shear-stress information in to spatial coordinates. This value was estimated (using two probes separated by $\delta$ in the streamwise direction) to be equal to $0.76 U_{\infty}$. This is within $15 \%$ of the previous estimates of the advection velocity in Hutchins et al. (2011), who measured this velocity using two probes separated by $0.7 \delta$. This difference in advection velocity does not significantly alter the filtered large-scale signal.

An example of instantaneous wall-shear-stress fluctuations, $u_{\tau}$, and streamwise velocity fluctuations, $u$, related to a low large-scale wall-shear-stress event and to a high large-scale wallshear-stress event are shown in figure 5. In figure $5 \mathrm{a}$, the footprint of a low wall-shear-stress event at the wall is highlighted by an extended negative region of the friction velocity fluctuations. In the velocity field associated with this low wall-shear-stress event, a large structure with negative values of the streamwise fluctuations is revealed. Similarly, for the high wall-shear-stress event, an elongated region that contains high momentum fluid (positive fluctuations) is apparent. This structure extends over several boundary layer thicknesses, from approximately $-2 \delta$ to $4 \delta$.

\subsection{Scale decomposition of the velocity field}

In order to look at the scale modulation during wall shear-stress event, the small and large-scale fluctuations are extracted from the PIV fields. First, the large-scale component of the velocity fluctuations, $u_{L}$ and $v_{L}$, are computed using a moving average box-filter that is $1 \delta$ long (this filter is only applied along the streamwise direction). The large-scale contributions are then subtracted from the total fluctuation to estimate the small-scale fluctuations, $u_{s}$ and $v_{s}$ directly from the PIV fields. This processing is illustrated in figure 6 . The length of the filter is the same as the one used for the wall-shear-stress signal and is consistent with the scale decomposition used by Hutchins and Marusic (2007b). A small change in filter length will have only a small 


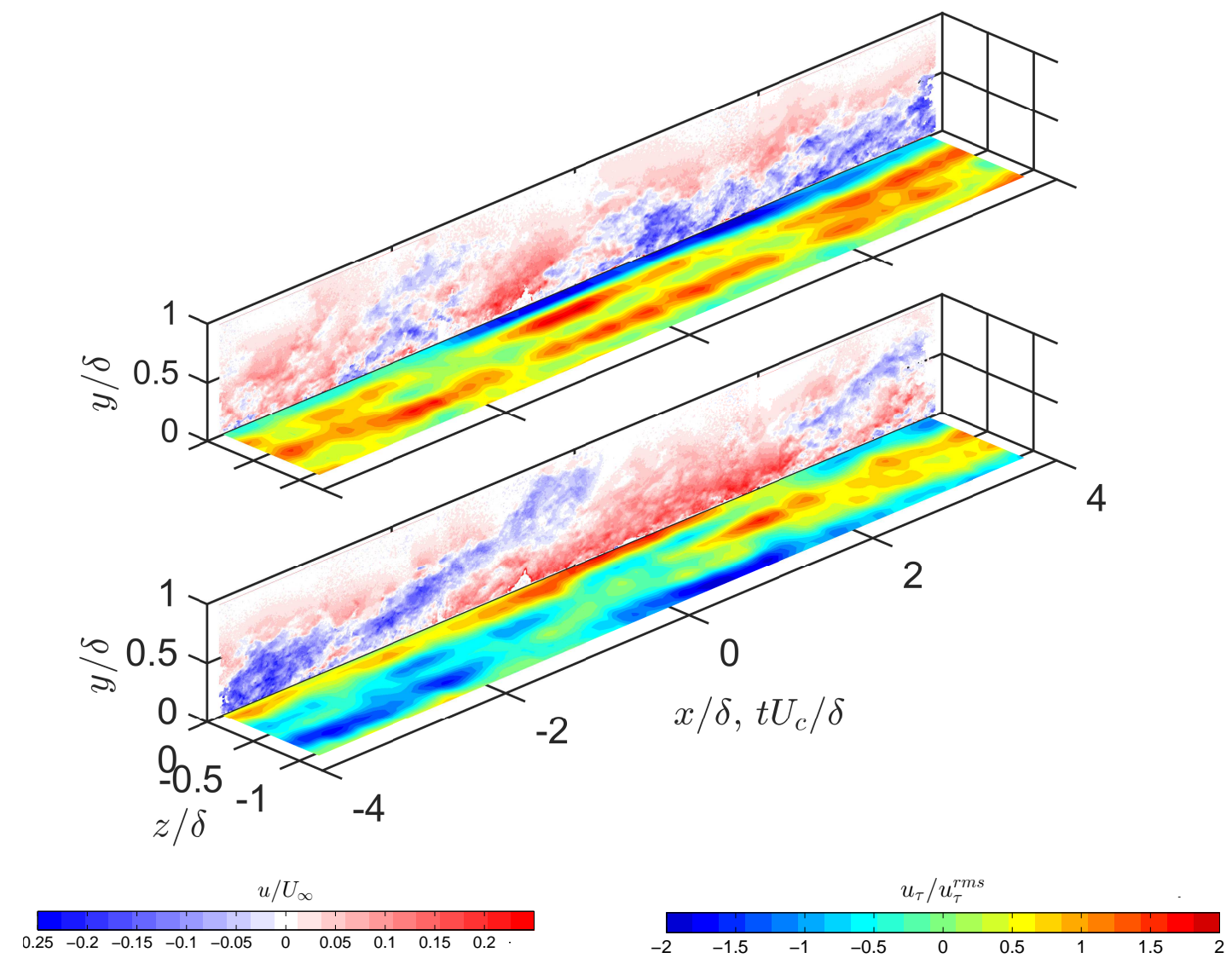

Figure 5: Instantaneous fluctuations of the friction velocity, $u_{\tau}$, at the wall and of the streamwise velocity, $u$, in the laser sheet plane associated with a low shear-stress event (top) and high shearstress event (bottom).

influence on the modulation analysis as discussed in Ganapathisubramani et al. (2012).

Now, this simultaneous data that captures both the footprint of very-large-scale motions at the wall as well as the its manifestation in the velocity field can be statistically characterized.

\section{Flow associated with large-scale wall-shear-stress events}

Hutchins et al. (2011) computed conditional averages of the flow based on the sign of the fluctuations of the friction velocity. For a negative wall-shear fluctuation, they observed an elongated low-speed region. This low speed region was approximately $0.3-0.5 \delta$ wide and is bordered by high-speed regions on both sides. The distance between the center of the high-speed regions is about $\delta$. The wall-normal velocity was found to be anti-correlated with the streamwise component and revealed the presence of large-scale counter-rotating roll-modes (Talluru et al. 2014). In case of a positive wall-shear-stress event, the flow structure differs only by the sign of the velocity fluctuation compared to a low wall-shear-stress event. Moreover, as described in several studies, 

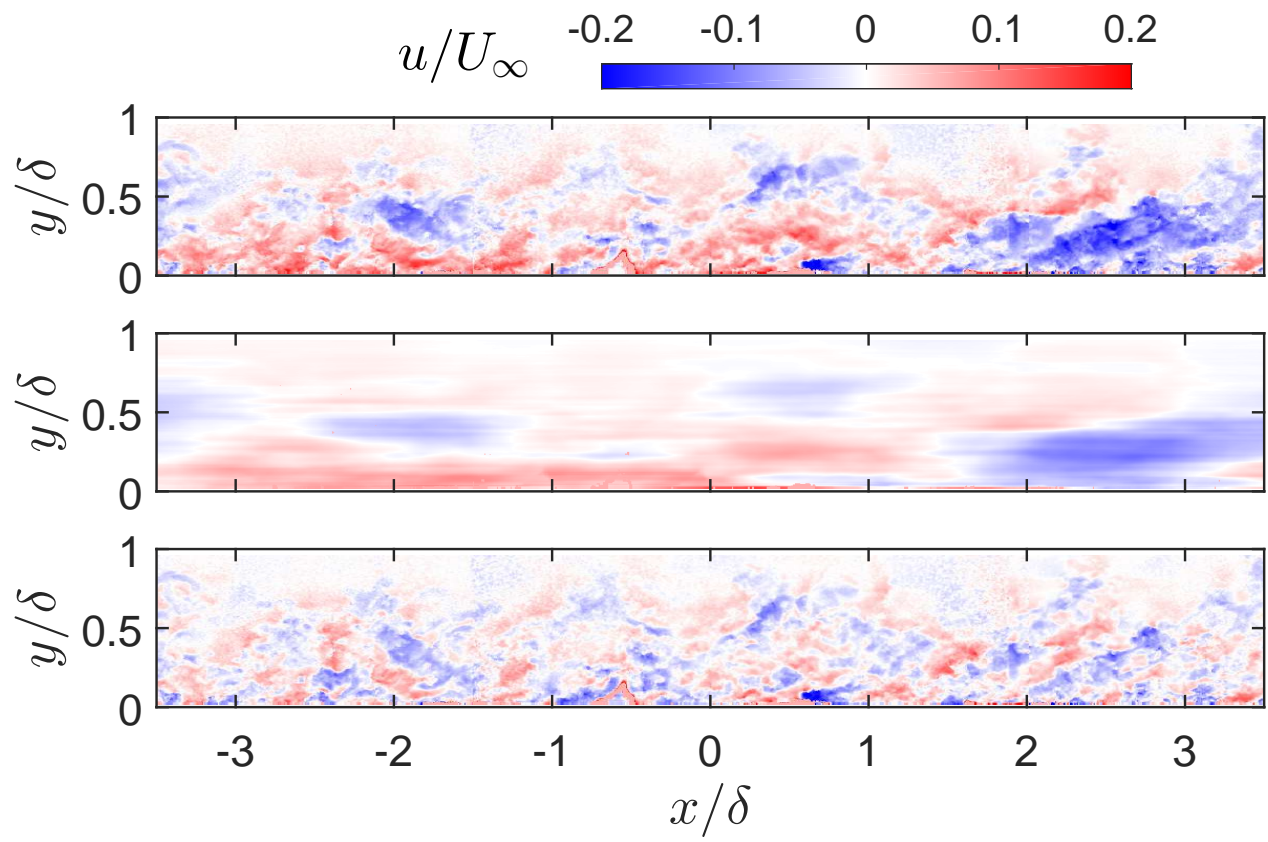

Figure 6: Decomposition of the streamwise velocity fluctuation: (top) Total streamwise fluctuation, $u$; (middle) Large-scale fluctuation, $u_{l}$, computed with moving average (box-filter) that is $1 \delta$ long; (bottom) Small-scale fluctuation computed by subtracting the field (top) and (bottom). 
including Hutchins et al. (2011), Ganapathisubramani et al. (2012) and Talluru et al. (2014), small-scale fluctuations are modulated by the presence of large-scale structures. For a negative wall shear-stress event, the small-scale fluctuations are reduced near the wall and are amplified away from the wall. Talluru et al. (2014) also shown that the unfiltered Reynolds shear-stresses appear to be modulated in a similar fashion as the small-scale velocity components.

In previous studies, only the sign of the friction velocity fluctuations was taken into account in the conditional average. In order to analyse the flow characteristics associated with largescale wall-shear-stress fluctuations, the histogram of the friction velocity fluctuations (figure 3 ) is divided into four quartiles, each containing $25 \%$ of the PIV fields. This corresponds to approximately 1000 occurrences in each quartile. Because the skewness of the histogram of the large-scale fluctuations at the wall is zero, the first two bins correspond to the negative part of the histogram, while the two other bins are associated with positive events. Given this decomposition of the histogram, the conditional averages of the conditional velocity fluctuations, $\langle u\rangle$ and $\langle v\rangle$ are evaluated. For the small-scale velocity components, instead of the average, the variance is computed, since, by definition, the average of the small-scale fluctuations is zero (as noted by Hutchins et al. (2011)). Therefore, the difference of the conditional small-scale variance and the time-average of the variance reflects the modulation of the small-scale by the large-scales. For example, the conditional variance above a negative wall-shear-stress event is then defined as:

$$
\left.\widetilde{\left\langle u_{s}^{2}\right.}(x, y, z)\right\rangle_{l}=\left\langle u_{s}^{2}\left(x, y, z_{0}\right) \forall u_{\tau}\left(x_{0}, z_{0}+\Delta z\right)<0\right\rangle-\overline{u_{s}^{2}}
$$

In a similar manner, the conditional unfiltered Reynolds shear-stress, $\langle-u v\rangle$, minus the time average, $\overline{-u v},\langle\widetilde{-u v}\rangle$ is computed.

Figure 7 shows the histogram of skin-friction velocity fluctuations together with results for conditional averages based on the quartiles. The quartiles in the histogram are color-coded with blue, green, orange, and red (lowest to highest value, figure 7a). Figures $7 \mathrm{~b}$ and $7 \mathrm{c}$ present the profiles of the conditional streamwise and wall-normal fluctuations, $\langle u\rangle$ and $\langle v\rangle$, at the mid-plane at a streamwise location $x / \delta=1$ (which is a location that is downstream of the wall-shear-stress array). This streamwise location is chosen as it shows the highest deviation in conditional mean velocity. These conditional profiles did not exhibit a significant change along the streamwise direction, however, the size of our ensemble (1000 velocity fields) led to small variations in the profile. Therefore, in order to achieve a better convergence of the profile, a running average of the conditional profile was computed. This running-average was computed over a streamwise distance of $1 \delta$. Therefore, for $x / \delta=1$, the profile is in fact averaged from $0.5 \delta$ to $1.5 \delta$. We note that this averaging length is the same as the length of the filter used for the decomposition between small-scales and large-scales and therefore will not significantly influence the results.

The conditional streamwise fluctuations associated with the extreme parts of the histogram (the solid blue and the red lines, respectively) show a large variation. At the peak of the profile, at approximately $y / \delta=0.1$, the extreme events account for $75 \%$ of the velocity deficit (or increase) associated with a negative (or positive) wall-shear-stress event. The profiles for the wall-normal fluctuation show similar results with a larger contribution of the extreme parts of the histogram in the change of the wall-normal fluctuations above a large-scale wall-shear-stress event. It is interesting to note that the largest amount vertical fluctuation (both positive and negative) is approximately at $y / \delta=0.2$. This suggest that the vertical transport of momentum (both ways, sweeps and ejections) should reach a maximum near this location (and this will be confirmed later in this section). The trends for the internal part of the histogram, dot-dashed (orange) and dashed (green) lines, collapse around $0.6 \delta$ while the curves for the extreme events appear to collapse towards the edge of the boundary layer. 


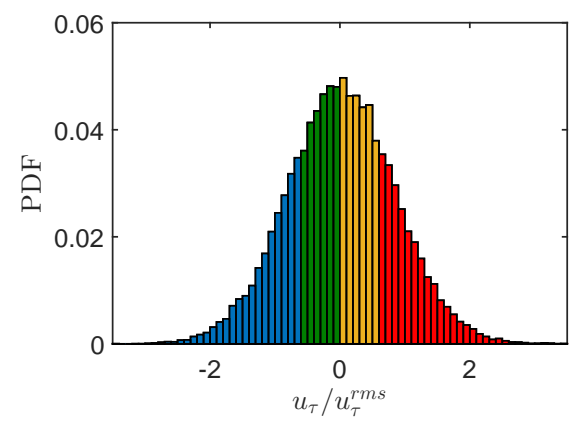

(a)

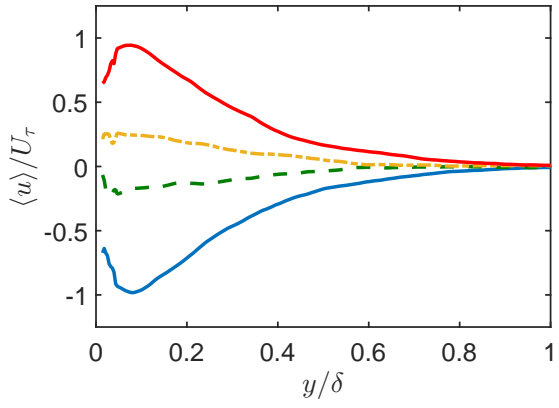

(b)

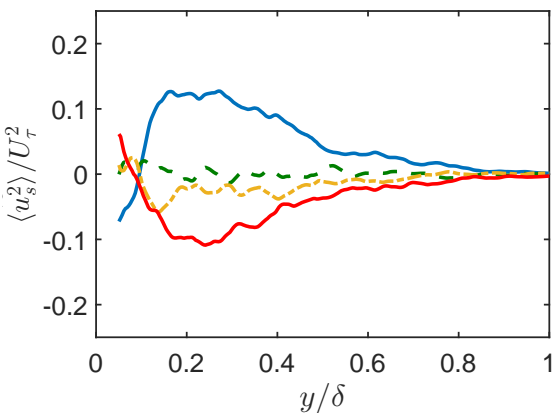

(d)

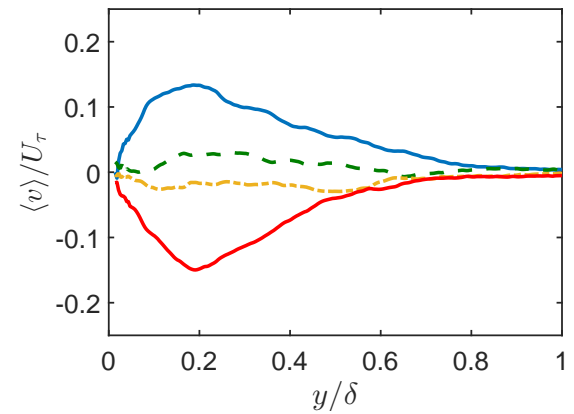

(c)

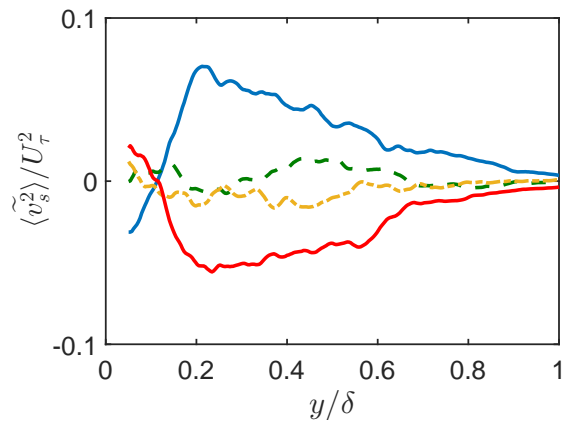

(e)

Figure 7: Extreme wall-shear-stress events: a) Decomposition of the histogram of the friction velocity fluctuations in four bins, each containing $25 \%$ of the occurrences b) Profiles of the conditional streamwise velocity fluctuation in the centerline $(z / \delta=0)$ at $x / \delta=1$ The color line corresponds to the part of the histogram of the same color. c) Profiles of the change of the small-scale variance of the streamwise velocity component. d) Profiles of the change of the small-scale variance of the wall-normal velocity component. Note that the conditional profiles were running-averaged from $0.5 \delta$ to $1.5 \delta$ in order to improve statistical convergence. 

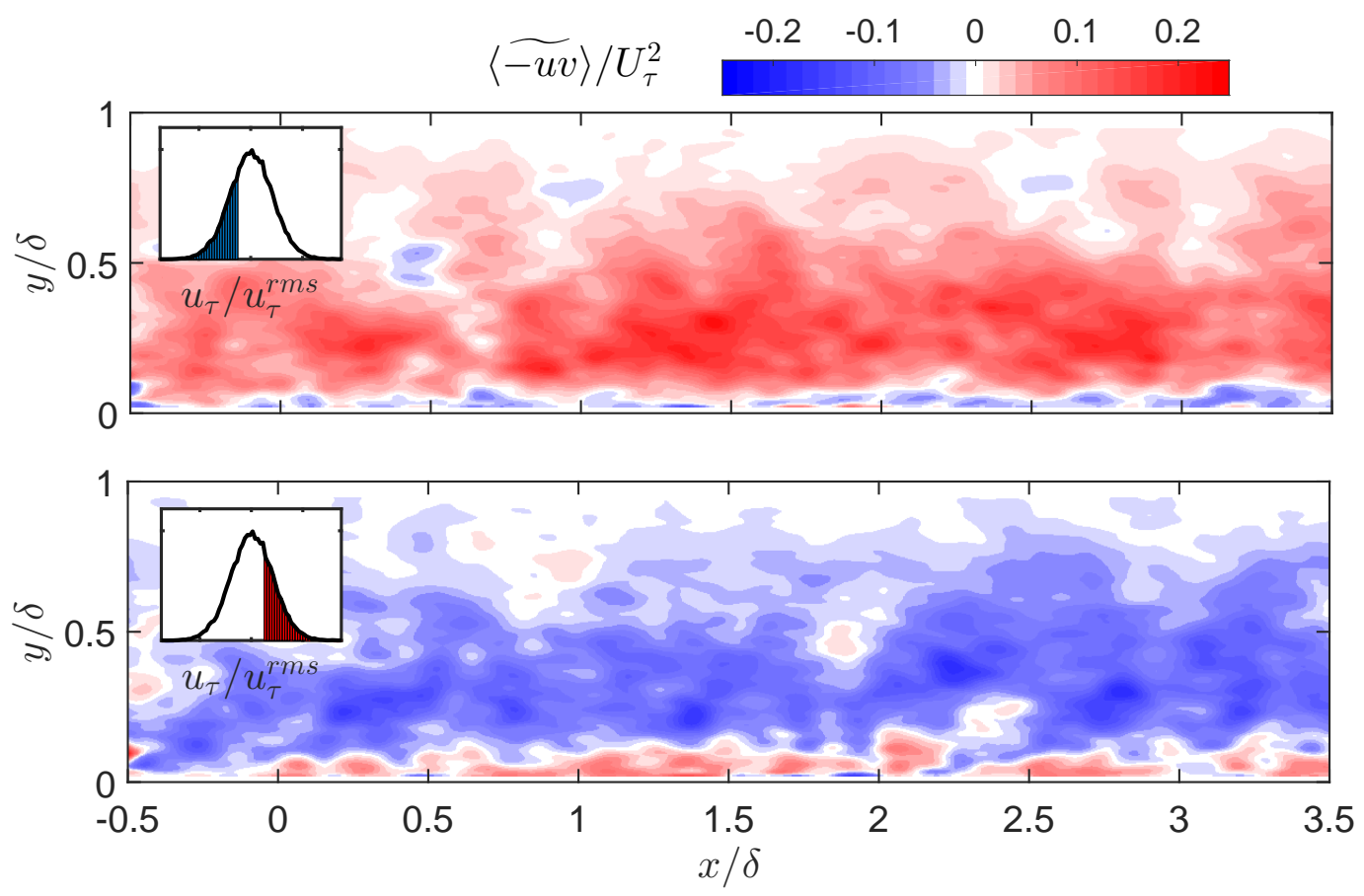

Figure 8: Change of the Reynolds shear-stress activity for the two extreme parts of the histogram in the plane $z / \delta=0$ : a) Field associated with a very low wall-shear-stress event b) Field associated with a very high wall-shear-stress event.

The small-scale conditional variance of streamwise and wall-normal velocity fluctuations are shown in figure $7 \mathrm{~d}$ and figure $7 \mathrm{e}$, respectively. It can be seen that the extreme events make a major contribution to the modulation of the small-scale activity, both in the near wall region and further away from the wall. The contribution from the central portion of the wall-stress histogram is not significant (as the values are close to the resolution of the velocity measurement). These observations indicate that the really strong (or extreme) events in wall-shear-stress are associated with the largest changes in the conditional velocity as well as the small-scale activity. Ideally, it would be desirable to decompose these extreme events with another condition. As a example, a simple additional condition we could impose is the existence time of the wall-shear-stress event. However, when this condition is imposed, the number of independent PIV vector fields that can be used for conditional averages reduces significantly and therefore renders that analysis impossible with the current dataset.

Figure 8 shows the conditional fields of the Reynolds shear-stress, $\langle\widetilde{-u v}\rangle$, related to the two extreme parts of the histogram, in the center plane $z / \delta=0$. Note that this Reynolds shearstress is in fact the difference between the conditional Reynolds shear-stress and the overall time-averaged Reynolds shear-stress. The red region corresponds to an increase of the Reynolds shear-stress activity and the blue area to a decrease of this activity relative to the time-averaged unconditional Reynolds shear-stress. This reveals that the global behaviour of the Reynolds shear-stress is reversed between these two contributions. For a low wall-shear-stress event, an attenuation of $\langle-u v\rangle$ near the wall and an increase away from the wall are observed. However, from this field representation, the amplitude of the increase of the Reynolds shear-stress activity 


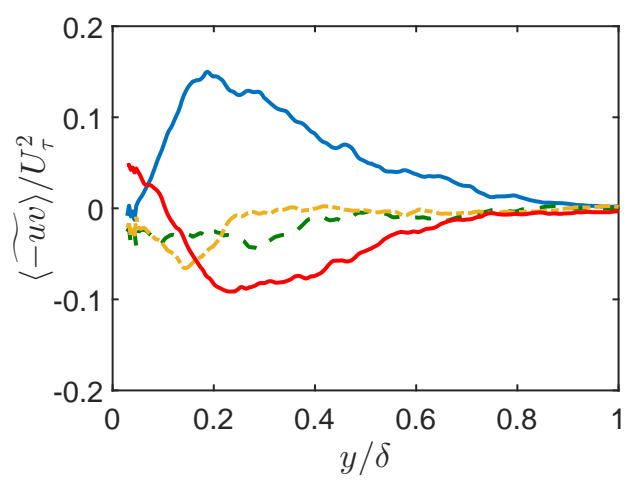

Figure 9: Profiles of the conditional Reynolds shear-stress in the middle plan $z=0$ and averaged between $0.5 \delta$ and $1.5 \delta$. The blue, green, orange and red correspond respectively to the very low shear, slightly low, slightly high and very high stress event at the wall given the histogram decomposition.

away from the wall seems to be higher than the decrease in the case of a high wall-shear-stress event. The modulation in the near wall region and the location of the crossover point for a extreme positive event appears to be lower compared to an extreme negative event. The limited size of our ensemble (1000 velocity fields) is responsible for the lack of convergence of this secondorder quantity as seen in the figure. However, the streamwise trend of the Reynolds shear-stress appears to be relatively unchanged and therefore, as before, the conditional Reynolds shearstress is averaged from $0.5 \delta$ to $1.5 \delta$ to compute the profiles and improve convergence. Again, we note that this averaging length is the same as the length of the filter used for the decomposition between small-scales and large-scales and therefore will not influence the results.

Figure 9 provides the profiles of the change in the Reynolds shear-stress activity, $\langle\widetilde{-u v}\rangle$ for the four bins of the histogram. This figure confirms the observations made in the previous field representation. In contrast with previous results associated only with the sign of the wallshear-stress event, the changes in the Reynolds shear-stress between symmetrical parts of the histogram are here not symmetric. Near the wall, the extreme negative event corresponds to a slight decrease of the Reynolds shear-stress compared to the time-average. However, for this part of the histogram, a strong increase of the Reynolds shear-stress in the outer region, with a peak around $0.2 \delta$ is observed. This is consistent with the peak in conditional vertical velocity fluctuation. For the very high wall-shear-stress events, i.e. the red part of the histogram in figure $7 \mathrm{a}$, an increase of the Reynolds shear-stress close to the wall is observed. Away from the wall, this contribution becomes negative but with a relatively low amplitude compared to the very low wall-shear-stress event. We can also note that the two intermediary parts correspond to negative contributions of the Reynolds shear-stress compared to the time-average. Accordingly, in the outer region of the boundary layer, the flow structures associated with the extreme negative wall-shear-stress events carry the largest amount of the Reynolds shear-stress. This is consistent with the fact that momentum flux from the wall should correspond to strong ejection events (where $u<0$ and $v>0$ ) that is predominantly associated with extreme large-scale negative skin-friction events (where $u$ is more negative in the near-wall region). 


\section{Scale decomposition of the Reynolds shear-stress}

To further examine the asymmetry in the conditional profiles of $\langle\widetilde{-u v}\rangle$, the Reynolds shearstress is decomposed using the small/large-scale decomposition presented in part 4.2. Based on this decomposition, the Reynolds shear-stress can be written in a similar form as in de Kat and Ganapathisubramani (2013), as the addition of four terms:

$$
u v=u_{l} v_{l}+u_{l} v_{s}+u_{s} v_{l}+u_{s} v_{s}
$$

Figure 10 provides the contribution of each term of the decomposition to the ensembleaveraged profile. The two cross-terms between large and small scales, $u_{l} v_{s}$ and $u_{s} v_{L}$, have a negligible contribution to the Reynolds shear-stress (dash line and dot line, respectively) compared to the two other terms. The profile associated with the small-scale, $\overline{-u_{s} v_{s}}$ (dashed dot line), reveals a peak close to the wall at $y / \delta=0.06$. The amplitude of this peak is similar to the term related to the large scale, $\overline{-u_{l} v_{l}}$ (solid line). However, for the latter, the maximum is located farther away from the wall, around $y / \delta=0.17$. The contribution of the large-scales is predominant in the outer region of the boundary layer.

This above observation can be used to speculate on the movement of the peak in Reynolds shear-stress with Reynolds number. The peak for the large-scale shear-stress seems to be located at the outer end of the logarithmic region while the peak for the small-scale shear-stress appears to be closed to the wall. There is a gradual increase and decrease of the two profiles with the crossover point occurring at around $\mathrm{y} \approx 0.07 \delta$. This point closely corresponds to the geometric center of the log region and is the location of cross-over for amplitude modulation as well as the location of peak Reynolds shear-stress. Therefore, the peak Reynolds shear-stress location can be viewed as the wall-normal location where the small-scales and large-scales have an equal contribution to the total Reynolds shear-stress. It must be noted that this observation is made with just one dataset at a single Reynolds number. Further data at different (but higher) Reynolds number would be required to confirm this observation.

Using the above described Reynolds shear-stress decomposition, the conditional average $\langle\widetilde{-u v}\rangle$ based on the 4 bins of the histogram is computed. Figure 11 presents the profiles of the Reynolds shear-stress in the middle plane $z / \delta=0$ (and streamwise-averaged between $0.5 \delta$ and $1.5 \delta$ ) for each bin of the histogram. In this figure, only the two predominant terms, $\left\langle-\widetilde{u_{s} v_{s}}\right\rangle$ and $\left\langle-\widetilde{u}_{l} v_{l}\right\rangle$, are plotted. The term associated with the small-scales, figure 11a, appears to exhibit symmetric trends for the positive and negative bins of the histogram. In particular, the two extreme parts, red and blue lines, associated with high amplitude wall-shear-stress events exhibit equal and opposite behaviour. It can also be seen from the profiles that the small-scale Reynolds shear-stress behaves in a similar fashion as the small-scale normal stresses, $\left\langle\widetilde{u_{s}^{2}}\right\rangle$ and $\left\langle\widetilde{v_{s}^{2}}\right\rangle$.

The large-scale Reynolds shear-stress, $\left\langle\widetilde{-u_{l} v_{l}}\right\rangle$, does not exhibit the symmetry in the profiles for the two extreme bins of the histogram. For a very low wall-shear-stress event, the solid (blue) line in figure 11b, a large increase of the large-scale term of the Reynolds shear-stress across the boundary layer is revealed. Close to the wall this term is close to zero and the maximum of the profile is located around $y / \delta=0.19$. This suggests that the large-scale structures are larger for an extreme negative shear-stress event and extends further in to the boundary layer. This structure also seems to an influence over a wider wall-normal range as seen in figure 11b. Based on this, it can be concluded that extreme negative shear-stress fluctuation event is accompanied by a large-scale structure that extends further from the wall (although does not significantly affect $\left.\delta_{99}\right)$ and its effect on large-scale Reynolds stress is significant. 


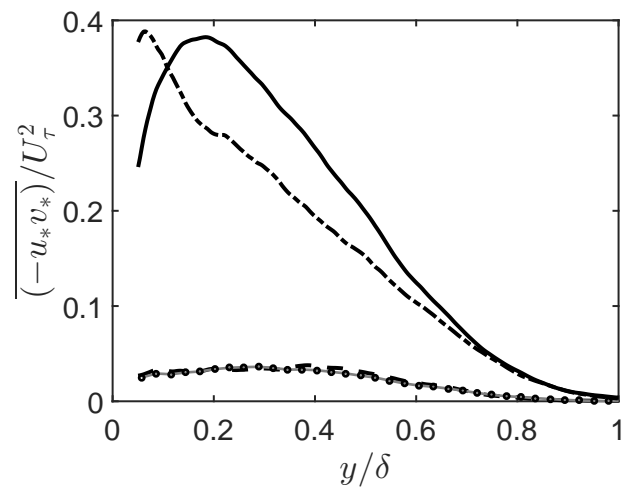

Figure 10: Reynolds shear-stress profile based on the large/small-scale decomposition : The solid line corresponds to $\overline{-u_{l} v_{l}}$, the dashed-dot line to $\overline{-u_{s} v_{s}}$, the dashed line to $\overline{-u_{l} v_{s}}$ and the triangles to $\overline{-u_{s} v_{l}}$

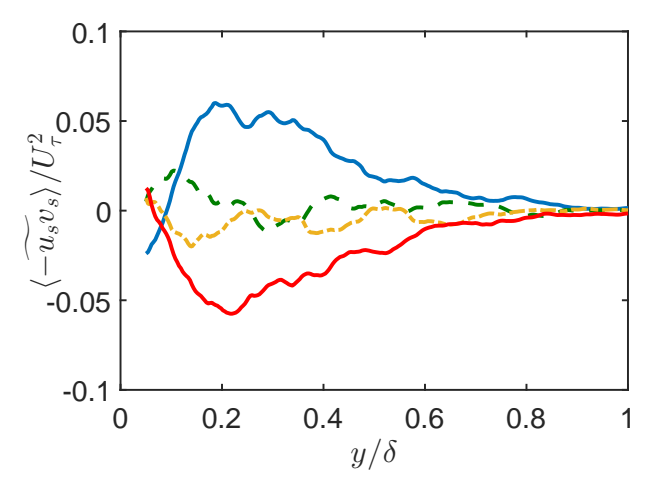

(a)

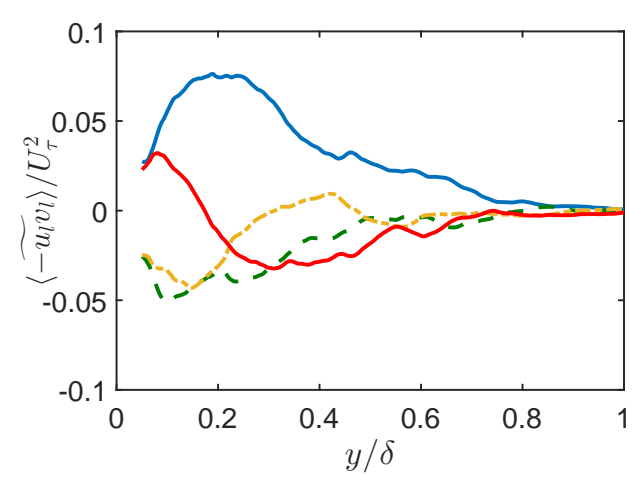

(b)

Figure 11: Change of the Reynolds shear-stress activity given the large/small-scale decomposition for the 4 bins of the histogram. Profile is located in the middle plan $z=0$ and averaged between $0.5 \delta$ and $1.5 \delta$. a) $\left\langle-\widetilde{u_{s} v_{s}}\right\rangle$ b) $\left\langle-\widetilde{u_{l} v_{l}}\right\rangle$ 
In the near wall region, the term $\left\langle\widetilde{-u_{l} v_{l}}\right\rangle$ associated with the very high wall-shear-stress fluctuation event, red line, has a similar trend as the low wall-shear-stress fluctuation event. However, beyond $y / \delta=0.08$, this term decreases and becomes negative after $y / \delta=0.18$. The two intermediary parts of the histogram, the dashed (green) line and the dot-dashed (orange) line, correspond to negative contributions of this large-scale term. Therefore, the difference in the Reynolds activity between the very low and high wall-shear-stress events is mainly due to the large-scale term $-u_{l} v_{l}$. This large-scale term contributes predominantly to the increase of the Reynolds shear-stress in the outer region of the boundary layer above a very low wall-shear-stress event. This asymmetry in the conditional averages is consistent with the recent observations of Agostini et al. (2016) who indicated that the processes are better captured if the scales are decomposes first based on sign and then on strength.

The above finding also has implications for using the FIK identity (Fukagata et al. 2002). This identity that shows that the mean wall-stress is related to the weighted average of the Reynolds shear-stress. Therefore, work has been carried out to determine the scale-by-scale contributions of the Reynolds shear-stress to the wall-stress(Deck et al. 2014). There might also be a tendency to extend this analysis to specific wall shear-stress events to identify associated physical mechanisms. The conditional averages presented herein has shown that a low shearstress event contributes to a larger proportion of the Reynolds shear-stress in the outer region compared to a high shear-stress events. Based on just the shear-stress information and the FIK identity, one might conclude that the this would in fact be a region of high shear-stress. Of course, the extent of the contribution will intimately depend on the filter scale and the type of decomposition. However, the data seems to indicate that a low shear-stress condition results in a high Reynolds shear-stress in the outer region. Consequently, care should be taken when applying integral methods such as the FIK identity to isolate skin-friction contributions from different physical mechanisms. In fact, Deck et al. (2014) indicated that the FIK identify is in fact a mathematical transformation and does not necessarily lead to physical reasonings. This is consistent with the findings of the current study where conditional averages computed based on physical motivation results in counter-intuitive trends in Reynolds shear-stress.

\section{Conclusion}

Simultaneous wide-field PIV and wall-shear-stress measurements have been performed to measure the large-scale structures associated with wall-shear-stress events in a high Reynolds number flat plate turbulent boundary $\left(R e_{\tau} \approx 4000\right)$. The combination of measurements allowed theflow, wall-normal and streamwise velocity components $(u$ and $v$ ) and of the Reynolds shear-stress $(-u v)$, related to a large-scale wall-shear-stress event. Moreover, the scale-decomposition of the velocity fluctuations allowed the analysis of the modulation of the small-scale activity by the large-scale structures.

Conditional averages computed based on strength of the wall-shear-stress event show marked differences for extreme positive and negative wall-shear-stress fluctuations. The histogram of the friction velocity fluctuations has been divided into four quartiles, each containing $25 \%$ of the occurrences. It has been shown that the $25 \%$ that contain the lowest or highest wall-shear-stress events contribute to $75 \%$ of the velocity deficit or surplus associated to negative or positive events. These extreme events contribute also predominantly in the modulation of the small-scale activity. Based on the results from skin-friction time signal analysis, it is hypothesized that these extreme events could be thought of as the central part of a much larger structure. It has been shown that the extreme negative wall-shear-stress event corresponds to a significant increase of the Reynolds shear-stress activity in the outer region of the boundary layer compared to the extreme 
positive event. The scale decomposition of the Reynolds shear-stress has demonstrated that this difference in the Reynolds shear-stress activity between very low and very high wall-shear-stress events are mainly due to the large-scale contribution $u_{l} v_{l}$.

It is worth noting that the analysis presented here has been limited to a condition on the strength of the wall-shear-stress event. We attempted to compute the above conditional averages with an addition size condition, however, this led to convergence issues with PIV data. Further studies with substantially larger amount of data is required to extend the analysis with multiple conditions.

\section{Acknowledgements}

The authors acknowledge the financial support of the European Research Council (ERC Grant agreement No. 277472), and the Engineering and Physical Sciences Research Council of the United Kingdom (EPSRC Grant Ref. No. EP/L006383/1). We are also grateful to Dr. Gregoire Fourrie for performing the shear-stress sensor measurements in collaboration with Dr. Roeland de Kat. All data supporting this study are openly available from the University of Southampton repository.

\section{References}

Agostini, L. and Leschziner, M. (2014). On the influence of outer large-scale structures on near-wall turbulence in channel flow. Physics of Fluids, 26(7):075107.

Agostini, L., Leschziner, M., and Gaitonde, D. (2016). Skewness-induced asymmetric modulation of small-scale turbulence by large-scale structures. Physics of Fluids, 28(1):015110.

Alfredsson, P. H., Johansson, A. V., Haritonidis, J. H., and Eckelmann, H. (1988). The fluctuating wall-shear stress and the velocity field in the viscous sublayer. Physics of Fluids (1958-1988), 31(5):1026-1033.

Bandyopadhyay, P. R. and Hussain, A. (1984). The coupling between scales in shear flows. Physics of Fluids (1958-1988), 27(9):2221-2228.

Bernardini, M. and Pirozzoli, S. (2011). Inner/outer layer interactions in turbulent boundary layers: a refined measure for the large-scale amplitude modulation mechanism. Physics of Fluids (1994-present), 23(6):061701.

Blackwelder, R. F. and Kovasznay, L. S. (1972). Time scales and correlations in a turbulent boundary layer. Physics of Fluids, 15:1545-1554.

Brown, G. L. and Thomas, A. S. (1977). Large structure in a turbulent boundary layer. Physics of Fluids (1958-1988), 20(10):S243-S252.

de Kat, R. and Ganapathisubramani, B. (2013). Characteristics of reynolds stresses in a turbulent boundary layer. In TSFP DIGITAL LIBRARY ONLINE. Begel House Inc.

Deck, S., Renard, N., Laraufie, R., and Weiss, P.-É. (2014). Large-scale contribution to mean wall shear stress in high-reynolds-number flat-plate boundary layers up to 13650. Journal of Fluid Mechanics, 743:202-248.

Del Alamo, J. C., Jiménez, J., Zandonade, P., and Moser, R. D. (2004). Scaling of the energy spectra of turbulent channels. Journal of Fluid Mechanics, 500:135-144. 
Eitel-Amor, G., Örlü, R., and Schlatter, P. (2014). Simulation and validation of a spatially evolving turbulent boundary layer up to $\operatorname{re} \theta=8300$. International Journal of Heat and Fluid Flow, 47:57-69.

Foucaut, J.-M., Carlier, J., and Stanislas, M. (2004). Piv optimization for the study of turbulent flow using spectral analysis. Measurement Science and Technology, 15(6):1046.

Fukagata, K., Iwamoto, K., and Kasagi, N. (2002). Contribution of reynolds stress distribution to the skin friction in wall-bounded flows. Physics of Fluids (1994-present), 14(11):L73-L76.

Ganapathisubramani, B., Hutchins, N., Monty, J., Chung, D., and Marusic, I. (2012). Amplitude and frequency modulation in wall turbulence. Journal of Fluid Mechanics, 712:61-91.

Ganapathisubramani, B., Longmire, E. K., and Marusic, I. (2003). Characteristics of vortex packets in turbulent boundary layers. Journal of Fluid Mechanics, 478:35-46.

Hutchins, N. and Marusic, I. (2007a). Evidence of very long meandering features in the logarithmic region of turbulent boundary layers. Journal of Fluid Mechanics, 579:1-28.

Hutchins, N. and Marusic, I. (2007b). Large-scale influences in near-wall turbulence. Philosophical Transactions of the Royal Society of London A: Mathematical, Physical and Engineering Sciences, 365(1852):647-664.

Hutchins, N., Monty, J., Ganapathisubramani, B., Ng, H., and Marusic, I. (2011). Threedimensional conditional structure of a high-reynolds-number turbulent boundary layer. Journal of Fluid Mechanics, 673:255-285.

Hutchins, N., Nickels, T. B., Marusic, I., and Chong, M. (2009). Hot-wire spatial resolution issues in wall-bounded turbulence. Journal of Fluid Mechanics, 635:103-136.

Jones, M., Marusic, I., and Perry, A. (2001). Evolution and structure of sink-flow turbulent boundary layers. Journal of Fluid Mechanics, 428:1-27.

Kähler, C., Stanislas, M., Dewhirst, T., and Carlier, J. (2002). Investigation of the spatiotemporal flow structure in the log-law region of a turbulent boundary layer by means of multi-plane stereo particle image velocimetry. In Laser Techniques for Fluid Mechanics, pages 39-53. Springer.

Kähler, C. J., Scharnowski, S., and Cierpka, C. (2012). On the uncertainty of digital piv and ptv near walls. Experiments in fluids, 52(6):1641-1656.

Kim, K. and Adrian, R. (1999). Very large-scale motion in the outer layer. Physics of Fluids (1994-present), 11(2):417-422.

Kovasznay, L. S., Kibens, V., and Blackwelder, R. F. (1970). Large-scale motion in the intermittent region of a turbulent boundary layer. Journal of Fluid Mechanics, 41(02):283-325.

Laskari, A., de Kat, R., and Ganapathisubramani, B. (2016). Full-field pressure from snapshot and time-resolved volumetric piv. Experiments in Fluids, 57(3):44.

Marusic, I., Mathis, R., and Hutchins, N. (2010). Predictive model for wall-bounded turbulent flow. Science, 329(5988):193-196.

Mathis, R., Hutchins, N., and Marusic, I. (2009). Large-scale amplitude modulation of the small-scale structures in turbulent boundary layers. Journal of Fluid Mechanics, 628:311-337. 
Schlatter, P. and Örlü, R. (2010). Assessment of direct numerical simulation data of turbulent boundary layers. Journal of Fluid Mechanics, 659:116-126.

Schlatter, P., Orlu, R., Li, Q., Brethouwer, G., Fransson, J. H., Johansson, A. V., Alfredsson, P. H., and Henningson, D. S. (2009). Turbulent boundary layers up to retheta $=2500$ studied through simulation and experiment. Physics of fluids, 21(5):51702.

Talluru, K., Baidya, R., Hutchins, N., and Marusic, I. (2014). Amplitude modulation of all three velocity components in turbulent boundary layers. Journal of Fluid Mechanics, 746:R1.

Tomkins, C. D. and Adrian, R. J. (2003). Spanwise structure and scale growth in turbulent boundary layers. Journal of Fluid Mechanics, 490:37-74.

Wark, C. E. and Nagib, H. (1991). Experimental investigation of coherent structures in turbulent boundary layers. Journal of Fluid Mechanics, 230:183-208.

Willert, C. E. and Gharib, M. (1991). Digital particle image velocimetry. Experiments in fluids, 10(4):181-193. 